\title{
Dynamics of slow and fast systems on complex networks
}

\author{
KAJARI GUPTA and G. AMBIKA* \\ Indian Institute of Science Education and Research, Pune 411 008, India \\ *Corresponding author. E-mail: g.ambika@iiserpune.ac.in
}

\begin{abstract}
We study the occurrence of frequency synchronized states with tunable emergent frequencies in a network of connected systems. This is achieved by the interplay between time scales of nonlinear dynamical systems connected to form a network, where out of $N$ systems, $m$ evolve on a slower time scale. In such systems, in addition to frequency synchronized states, we also observe amplitude death, synchronized clusters and multifrequency states. We report an interesting crossover behavior from fast to slow collective dynamics as the number of slow systems $m$ increases. The transition to amplitude death is analyzed in detail for minimal network configurations of 3 and 4 systems which actually form possible motifs of the full network.
\end{abstract}

Keywords. Complex networks; slow and fast systems; frequency synchronization; amplitude death.

PACS Nos $\quad$ 05.45.Yv; 05.45.Xt; 05.45.Gg; 89.75.Fb

\section{Introduction}

Many complex systems that occur in physical, biological, chemical and geophysical contexts have a large number of sub systems or units interacting with each other. The collective behavior of such highly interconnected dynamical systems is being studied recently using the framework of complex networks with the sub units represented by the nodes and the connectivity among them indicated by links. The varied levels of their complexity arise from the nonlinearity of the nodal dynamics, nonlinear or stochastic processes that happen on them and the nature of the interactions reflected in the complex topology of the networks. Some of the well studied emergent phenomena in such contexts are synchronization [1], amplitude death [2], clustered states and chimera states $[3,4]$.

Recent studies indicate that heterogeneity plays a major role in the emergent dynamics of such real-world complex networks. Heterogeneity can arise in complex networks in various ways, such as degree heterogeneity, heterogeneity in the weights or nature of coupling, parameter mismatch and dissimilar nodal dynamics, dynamical or time varying couplings. This can affect processes happening on the network like rumor or epidemics spreading $[5,6]$ ecosystem stability in ecological networks $[7,8]$ and plays an important role in the diversity and organization of social networks [9] or breaking synchrony in oscillator networks [10]. In power grids and the Internet it has been shown that heterogeneity due to different loads on nodes can cause a cascade effect of overload failures [11].

In this work, we consider the consequences of heterogeneity caused by different time scales of the nodal dynamics on a complex network. We note that this is highly prevalent in many complex systems and dynamical processes such as modulated lasers and chemical reactions $[12,13]$. Also in biological systems, dynamical processes with time scales of days interact with those of biochemical reactions with sub-second time scales [14]. In neuronal networks, electric signals of neurons get directly or indirectly affected by intercellular processes of various time scales [15]. In the context of weather and climate systems, sub systems of widely varying time scales get strongly coupled to each other, e.g. tropical atmospheric ocean systems $[16,17]$. It is reported that climate sensitivity and stabilization occur through processes with a range of time scales [18, 19]. There are also studies on populations with time-scale diversity [20] and spatio-temporal chaos with cascade of bifurcations caused by interactions among different time scales [21].

Recently, a basic study on coupled nonlinear systems with fast and slow time scales reported interesting emergent states like amplitude death state and frequency synchronized states [22]. However, a detailed 
analysis of nodal dynamics with different time scales has not been done in the context of complex networks. We find there are many interesting open questions to be addressed regarding the possible emergent dynamics, its characterization and transitions when nodal systems follow multiple time scales.

In this study, we present the emergent collective behavior in a network of nonlinear dynamical systems, where the heterogeneity arises only from the difference in the time scales of nodal dynamics. To make this a specific feature and bring out effects of time-scale mismatch of connected systems, we consider an otherwise homogeneous network topology of a fully connected network of $N$ systems in which $m$ evolve at a slow time scale compared to others. We observe dynamical states like synchronized clusters, multifrequency states, phase synchronized states, and phenomena like amplitude death and crossover behavior in the collective dynamics as $m$ is varied for different values of mismatch of time scales and coupling strength.

\section{Network of slow and fast systems}

We construct a network of $N$ identical $n$-dimensional systems in which $m$ evolve on a slower time scale. This subset of slow oscillators is defined as $\mathrm{S}$. The equation governing the dynamics of the $i^{\text {th }}$ node is given by

$\dot{X}_{i}=\tau_{i} F\left(X_{i}\right)+G \epsilon \tau_{i} \sum_{j=1}^{N} A_{i j}\left(X_{j}-X_{i}\right)$,

where $\tau_{i}=\tau$ if $i \in \mathrm{S}, \tau_{i}=1$ otherwise. $G$ is an $n \times n$ matrix that decides which variables are to be coupled. Here we take $G=\operatorname{diag}(1,0,0$..) which means that the $x$ variable of the $i^{\text {th }}$ oscillator is coupled diffusively with the $x$ variable of the $j^{\text {th }}$ oscillator. $A_{i j}$ is the adjacency matrix of the network defining its topology or connectivity. Since we consider a fully connected network, $A_{i j}=1$ for all $i$ and $j$ except $i=j$. In this study, we consider the dynamics at each node as a periodic Rössler system given by

$\dot{x}_{i}=\tau_{i}\left(-y_{i}-z_{i}\right)+\tau_{i} \epsilon \sum_{j=1}^{N} A_{i j}\left(x_{j}-x_{i}\right)$

$\dot{y}_{i}=\tau_{i}\left(x_{i}+a y_{i}\right)$

$\dot{z}_{i}=\tau_{i}\left(b+z_{i}\left(x_{i}-c\right)\right)$.

The parameters are chosen as $a=0.1, b=0.1$ and $c=4$ so that dynamics is in the periodic region. The frequency of the oscillations of the intrinsic system depends on the time-scale parameter $\tau$ and decreases linearly as $\tau$ is decreased. However, when the systems

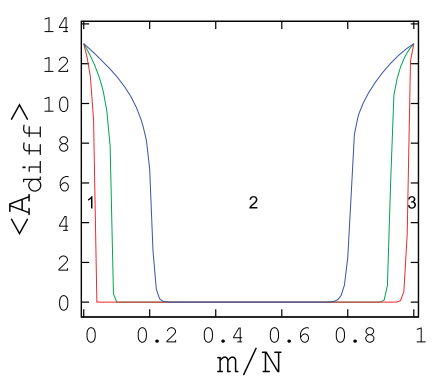

Figure 1. Average difference in amplitudes, $\left\langle A_{\text {diff }}\right\rangle$, of $N$ coupled Rössler systems plotted against the fraction, $m / N$ of slow systems. Here $N=100, \epsilon=0.03$ and $\tau=0.1$ (red), 0.35 (green), 0.5 (blue). Region 2 , where $\left\langle A_{\text {diff }}\right\rangle=0$ corresponds to AD.

are coupled to form the network, the emergent frequency may depend also on the number of slow systems $m$.

One of the main results of our study is the suppression of dynamics in the whole network, or amplitude death (AD) that occurs for sufficient time-scale mismatch and coupling strength for a range of values of $m$. To identify this range, we calculate the difference between global maxima and global minima, $\left(A_{\text {diff }}\right)$, in the $x$-time series of each system. The average of this difference is plotted for different $m$ values in a network of 100 systems in figure 1 . The region for which $\left\langle A_{\text {diff }}\right\rangle=0$ corresponds to suppression of oscillations or amplitude death (AD) marked as region 2. For values of $m$ corresponding to regions 1 and 3 we observe dynamical states like synchronized clusters. We discuss these phenomena in detail in the following section.

\subsection{Synchronized clusters: Multifrequency states and frequency synchronization for small $m$}

In the region 1 of figure 1 , where the number of slow systems $m$ is small (say $m \approx 10$ ), under sufficiently strong coupling, the whole network splits into clusters of slow and fast systems. The dynamics within each cluster is synchronized while the two clusters show frequency synchronized oscillations separated by a phase shift. This is indicated by the time series in figure 2 for $\tau=0.5$, $\epsilon=0.03$. We note that the amplitude of the cluster of fast systems is larger than that of the slow one. When the time-scale mismatch increases, this difference in amplitudes also increases.

The synchronization within each cluster is confirmed by calculating the variance of all oscillators in that cluster. The $v a r_{s}$ and $v a r_{f}$ thus calculated for slow and fast clusters respectively are shown in figure 3.

In this case, for very weak coupling $(\epsilon \approx 0.001)$ and large $\tau \approx 0.08$ we observe that the dynamics within the cluster of slow systems is in a two-frequency state of 


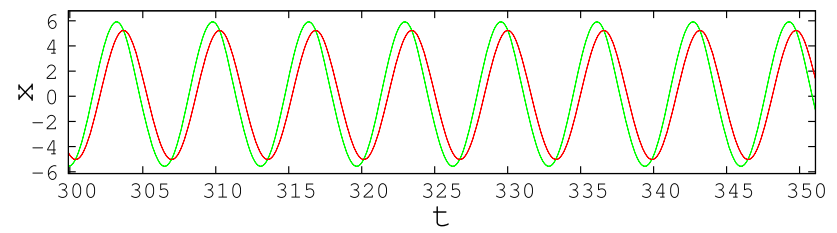

Figure 2. Synchronized clusters of slow and fast dynamics in the network for $\tau=0.5, \epsilon=0.03, m=10$ and $N=100$. Here the time series of the $x$ variables are plotted for 3 typical slow(red) and 3 fast(green) systems.
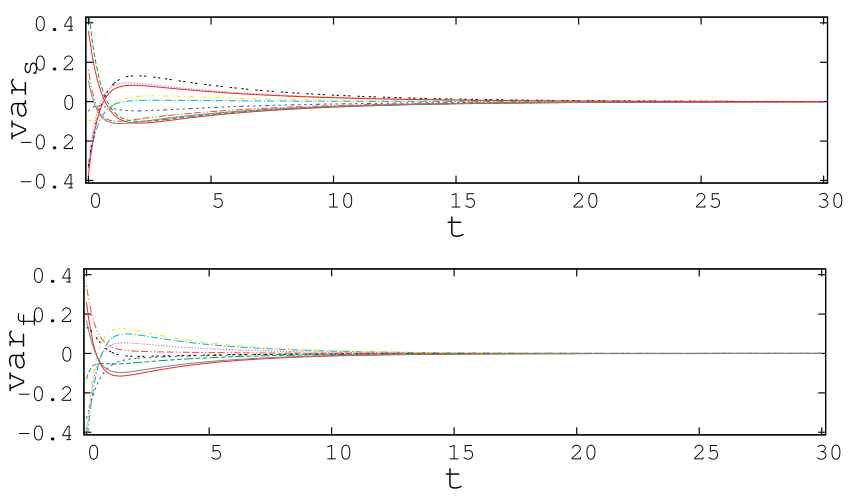

Figure 3. Variance of slow oscillators $\operatorname{var}_{s}$ and that of fast oscillators $v a r_{f}$ are plotted against time for $\tau=0.5$, $\epsilon=0.03$. After transients, $v a r_{s}=0$ and $v a r_{f}=0$, indicating that the dynamics within each cluster is completely synchronized.

small amplitude oscillations, while that in the other cluster is single frequency oscillations of large amplitudes (figure 4). Here also the dynamics within each cluster is completely synchronized.

\subsection{Suppression of dynamics and frequency synchronization for moderate $m$}

As the number of slow systems $m$ increases, corresponding to region 2 in figure 1 , mismatch in time scales of interacting slow and fast systems causes the suppression of dynamics in the whole network, observed for a specific range of $\tau$ and $\epsilon$. This state of AD is clear from figure 5 .

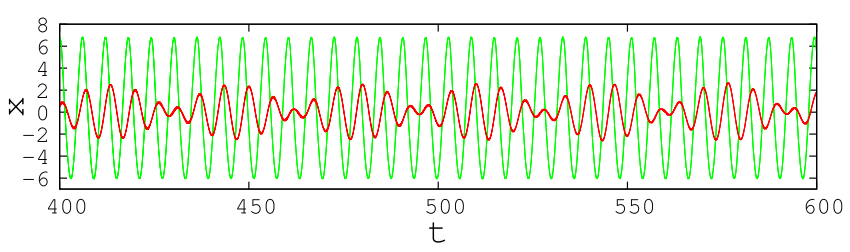

Figure 4. Time series of $x$ variables are plotted for 3 slow and 3 fast systems. This plot shows a two-frequency state for slow (red) systems, while fast (green) systems show large amplitude oscillations of single frequency. Here $\tau=0.8$, $\epsilon=0.001, m=10$ and $N=100$.

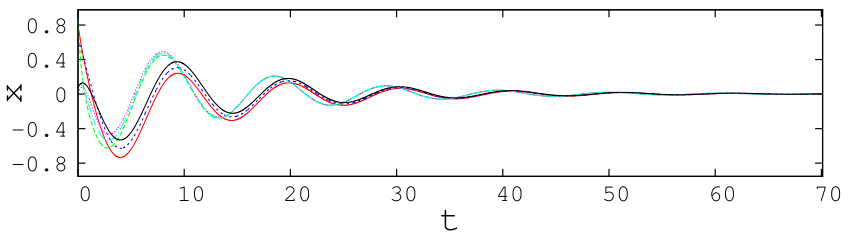

Figure 5. Amplitude death state for fully connected network of slow and fast systems for $\tau=0.35, \epsilon=0.03, m=50$, $N=100$. Here the time series of $x$ variable of 3 fast and 3 slow systems are plotted.

For any value of $m$ in Region 2 of figure 1, we can isolate the region of $\mathrm{AD}$ in $(\tau, \epsilon)$ parameter plane. For this we numerically calculate the average difference between global maxima and global minima $\left(A_{\text {diff }}\right)$ of all systems in the network and mark the region where this is zero. The region of $\mathrm{AD}$ thus obtained is shown in red in figure 6.

Outside the region of amplitude death, for weak coupling and large $\tau$, the systems go into two-frequency states as shown in figure 7 . In this case also, slow systems and fast systems are synchronized within each cluster. The two clusters have equal values for the smaller of the two emergent frequencies. For all the cases of synchronized clusters mentioned above, synchronization is verified by calculating the variances that behave qualitatively similar to figure 3 .

As the coupling strength increases we observe that there is a large region in the parameter plane where the whole network is frequency synchronized, but with a phase shift between the two clusters. This is qualitatively similar to the dynamics shown in figure 2 . We calculate the frequency of this emergent state from the time series, as reported in the earlier work [22]. The dependence of this frequency on the parameters $\epsilon$ and $\tau$ is clear from figure $8 \mathrm{a}$. In figure $8 \mathrm{~b}$ the emergent frequencies are compared with the intrinsic frequencies as $\tau$ is varied. For this range of $\tau$ and $\epsilon$, the emergent frequencies are less than the average of intrinsic fast and slow frequencies indicating frequency suppression.

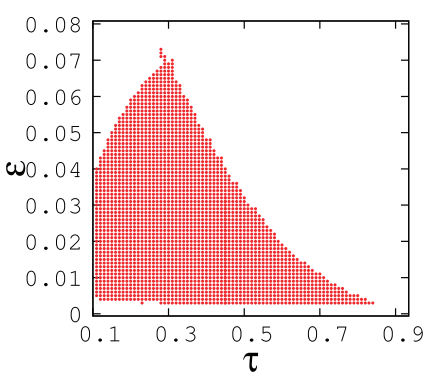

Figure 6. Region of amplitude death in $(\tau, \epsilon)$ plane for $m=50$ and $N=100$. 


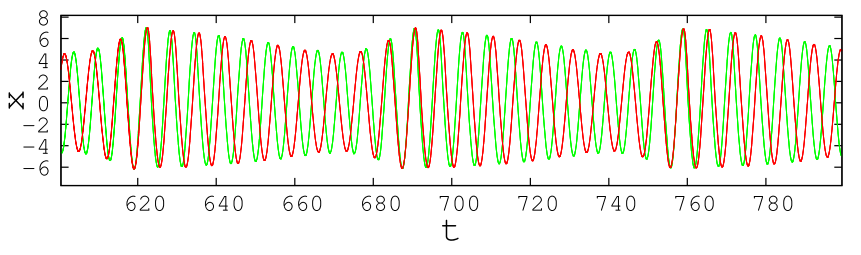

Figure 7. Time series of $x$ variables are plotted for 3 slow and 3 fast systems out of 100 systems in the network. This shows two-frequency states of slow (red) and fast (green) systems synchronized within the clusters for $m=50, \tau=0.9$ and $\epsilon=0.001$.
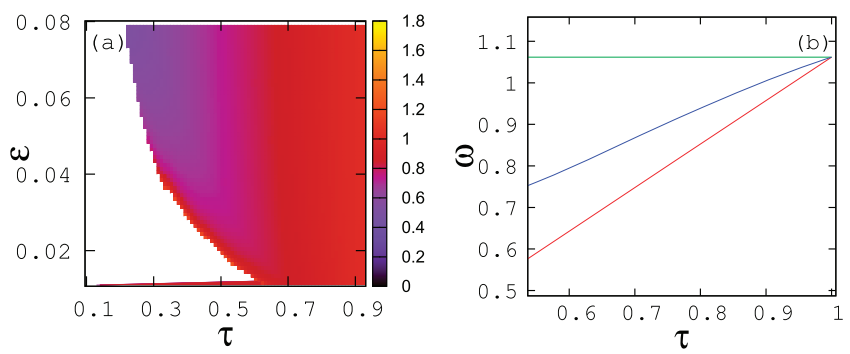

Figure 8. (a) Variation of the emergent frequency in $(\tau, \epsilon)$ plane for $m=50$ in the frequency synchronized state. The color code is as per the value of the frequency of the emergent state. (b) Variation of intrinsic frequency of fast systems (green) and that of slow systems (red) compared with the emergent frequency (blue) as $\tau$ is varied for $\epsilon=0.03$ and $m=50$.

\subsection{Crossover behaviour in dynamics for large $m$}

As $m$ increases further, corresponding to region 3 of figure 1 , the network in general regains the dynamics from the amplitude death state but follows a slower time scale. When the mismatch is not large or for large $\tau$, the systems form synchronized clusters. But in this case the slow systems have larger amplitude than the fast systems. This is a clear indication of a crossover behavior in the collective dynamics of the network as $m$ varies. This can be traced in two ways: by the emergent frequency of the frequency synchronized states and by the average amplitude of the synchronized clusters.

We observe that the emergent frequency in the frequency synchronized state decreases with the increase of $m$, as shown in figure 9a for a particular value of $\tau$ and $\epsilon$. Thus the frequency becomes less than the average of intrinsic fast and slow frequencies at some value of $m$, say $m_{1}$. This value of $m_{1}$ gives the critical value where frequency suppression sets in.

We also observe another crossover behavior in the amplitudes of oscillations of slow and fast systems when $m$ is increased. To study this, we plot the average amplitudes of the fast cluster and slow cluster in figure 9. The
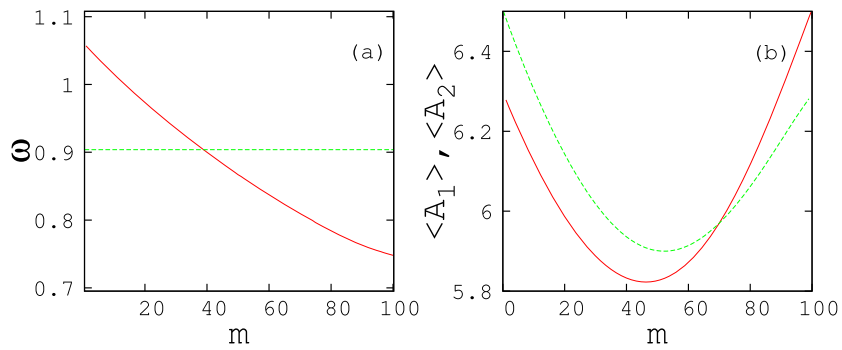

Figure 9. (a) Variation of emergent frequency with $m$ for $\epsilon=0.03$ and $\tau=0.7$ (shown in red). Green line shows the average of intrinsic fast and slow frequencies. Here frequency suppression happens for $m$ greater than $m_{1}=38$ at which red and green line intersect. (b) Average amplitude of slow (red) and fast (green) oscillators with $m$, showing crossover behavior at $m_{2}=70$ for $\epsilon=0.03$ and $\tau=0.7$.

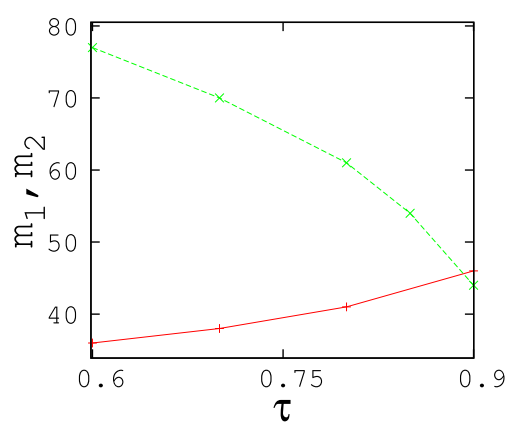

Figure 10. Variation of crossover thresholds with $\tau$ for $\epsilon=0.03$. Red line denotes the critical number of slow systems $\left(m_{1}\right)$ at which frequency suppression starts and green line denotes the same $\left(m_{2}\right)$ for crossover in amplitudes.

crossover from a state where the amplitudes of the fast cluster are larger, to one where those of the slow cluster are larger, happens at a value of $m$, say $m_{2}$, where the amplitudes cross each other. We find that both these crossover points vary with the parameters $\tau$ and $\epsilon$. To illustrate this, we plot $m_{1}$ and $m_{2}$ for $\epsilon=0.03$, with different values of $\tau$ (figure 10).

\section{Suppression of dynamics in minimal networks with differing time scales}

In this section, we analyze in detail the onset of amplitude death due to difference in time scales in two minimal configurations of the network with 3 and 4 systems each. These serve as possible motifs in the large network and can be subjected to analytical study of transition to AD. Such motifs are significant as repeating sub-graphs in any network. They represent basic interaction patterns that recur throughout the network [23, 24, 25].

We consider in figure 11, all possible configurations for $N=3$ and $N=4$ with two different time scales marked as S (slow) and F (fast). As we can see, for 


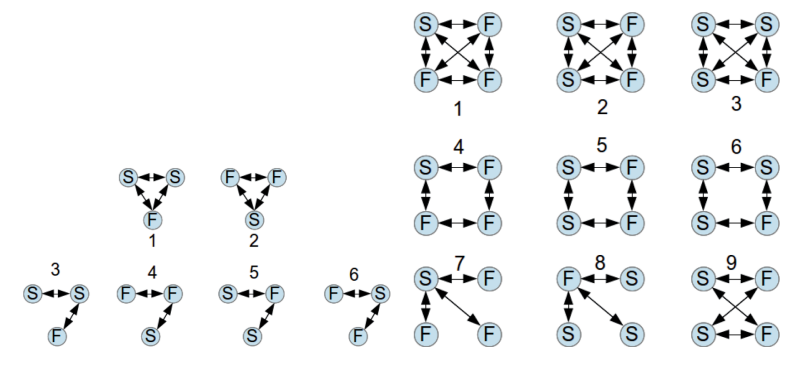

Figure 11. Configurations of minimal networks with 3 systems and 4 systems having different possible $m$.

$N=3$, the total number of unique configurations are 6 when $m$ is 1 or 2 . In the case of 4 systems, we have 9 configurations, ring, bipartite and fully connected structures, with $m=1,2$ and 3 .

The AD in this context corresponds to a common fixed point of the system and hence its onset and occurrence can be derived from the stability analysis of the fixed point of the system $\left(x^{*}, y^{*}, z^{*}\right)$. For this we calculate the eigenvalues of the Jacobian of each configuration, around the fixed point. The values of $\tau$ and $\epsilon$ for which all eigenvalues have negative real parts correspond to stable regions of $\mathrm{AD}$. The generic form of the Jacobian of any network of slow and fast systems can be written as

$\mathbf{J}=(\tau . \mathbf{I}) \mathbf{x} \mathbf{F}+(\tau . \mathbf{A}) \mathbf{x H}$,

where $\tau$ is an $N \times N$ matrix in which $\tau_{i j}$ corresponds to $\tau_{i}$ of eq. (1) for all $j$. $\mathbf{I}$ is $N \times N$ identity matrix. Dot product (.) is defined by the element-wise product of two matrices, and cross product (x) is defined as each element of the former matrix being multiplied by the latter matrix as a block. A is the adjacency matrix. In the case of Rössler systems with coupling function as given in eq. (2), we have

$\mathbf{F}=\left(\begin{array}{ccc}-k \epsilon & -1 & -1 \\ 1 & a & 0 \\ z * & 0 & (x *-c)\end{array}\right), \quad \mathbf{H}=\left(\begin{array}{lll}\epsilon & 0 & 0 \\ 0 & 0 & 0 \\ 0 & 0 & 0\end{array}\right)$

$\mathbf{0}=\left(\begin{array}{lll}0 & 0 & 0 \\ 0 & 0 & 0 \\ 0 & 0 & 0\end{array}\right)$

Here,

$$
\begin{aligned}
\left(x^{*}, y^{*}, z^{*}\right)= & \left(\frac{c-\sqrt{c^{2}-4 a b}}{2}, \frac{-c+\sqrt{c^{2}-4 a b}}{2 a},\right. \\
& \left.\frac{c-\sqrt{c^{2}-4 a b}}{2 a}\right),
\end{aligned}
$$

$k$ is the number of non-zero elements in the $i^{\text {th }}$ row of $\mathbf{A}$. For example, in the case of $N=4$, the Jacobian of the configuration 9 in figure 11 can be written as

$J=\left(\begin{array}{cccc}\tau \mathbf{F} & \mathbf{0} & \tau \mathbf{H} & \tau \mathbf{H} \\ \mathbf{0} & \tau \mathbf{F} & \tau \mathbf{H} & \tau \mathbf{H} \\ \mathbf{H} & \mathbf{H} & \mathbf{F} & \mathbf{0} \\ \mathbf{H} & \mathbf{H} & \mathbf{0} & \mathbf{F}\end{array}\right)$.

Here $k=2$ in the matrix $\mathbf{F}$ because $\mathbf{A}$ has 2 entries of 1 in each row.

We now estimate the eigenvalues of the Jacobian for each configuration for different values of $\tau$ and $\epsilon$ and identify the transition to $\mathrm{AD}$ as the point where the real part of at least one eigenvalue goes from negative to positive. This is repeated for all the configurations of $N=3$ and $N=4$, and the transition curves obtained in the $\tau, \epsilon$ plane are shown in figures 12 and 13. In these figures, the top left part of the transition curve indicates transition to instability, while the other two curves correspond to transition from amplitude death to oscillations.

We observe that in general the transition curve depends primarily on the number of slow systems $m$. For the same $m$ the configurations which are all to all and bipartite have identical transition curves. Thus, for $N=3$ and $m=1$, configurations 1 and 5 have identical transitions (figure 12a). In this case, of the nine eigenvalues of the Jacobian, six are common. At the transition the real part of one of the common complex conjugate pair crosses zero for the same set of parameter values indicating Hopf bifurcation. Similar results are obtained for configurations 2 and 6 with $m=2$ (figure 12b). However configurations 3 and 4 with different $m$ show different transition curves (figure 12c).

Similarly for 4 systems, each pair of configurations 1 and $7(m=1), 2$ and $9(m=2)$ and 3 and $8(m=3)$ has the same transition curves as shown in figures $13 \mathrm{a}, \mathrm{b}$ and $\mathrm{c}$. Here each pair has all to all and bipartite structures, and an equal pair of eigenvalues cross at the transition just like the case of 3 systems. We find that configurations 4 , 5 and 6 with different $m$ and ring topology show different transition curves (figure 13d).
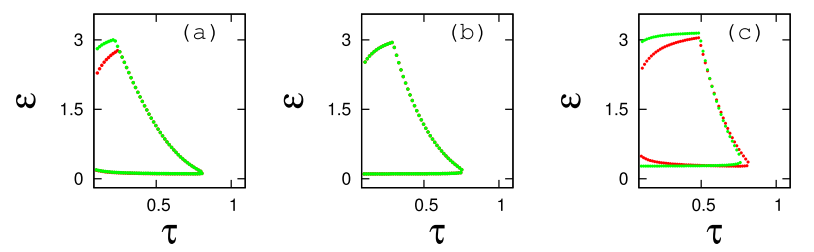

Figure 12. Transition curves for $\mathrm{AD}$ with 3 systems for configurations (a) 1 (red) and 5 (green), (b) 2 (red) and 6 (green) showing fully connected and bipartite cases with identical transitions, (c) 3 (red) and 4 (green) with different transition curves. 

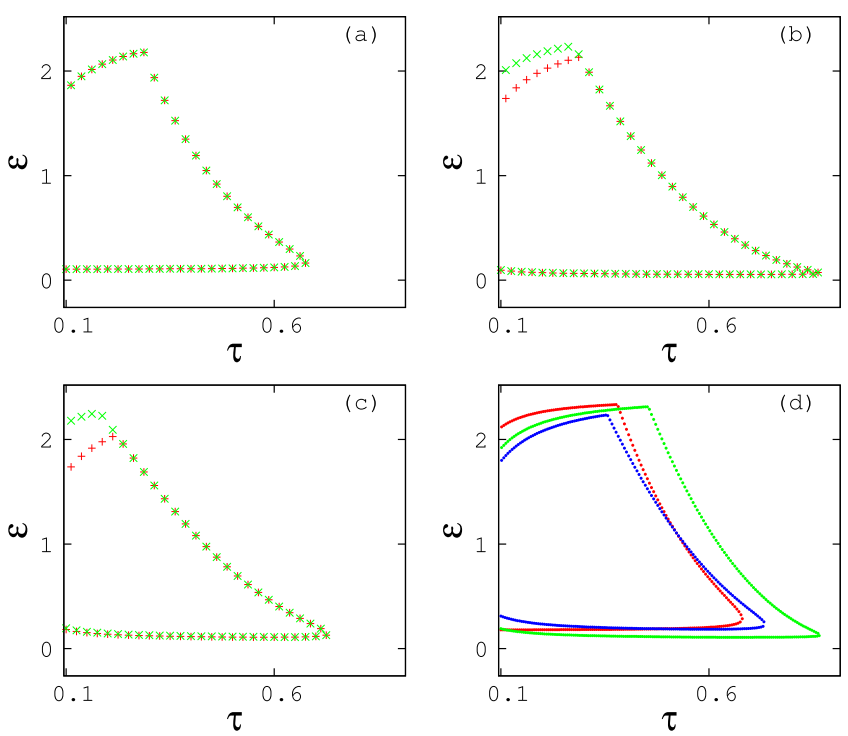

Figure 13. Transition curves for AD with 4 systems for configurations (a) 1 (red) and 7 (green) (b) 2 (red) and 9 (green) (c) 3 (red) and 8 (green) showing fully connected and bipartite cases with identical transitions, (d) 4 (red), 5 (green) and 6 (blue) with different transition curves.

Outside the region of amplitude death we see clusters of slow and fast systems for fully connected and bipartite configurations in both $N=3$ and 4 cases. The clusters are frequency synchronized with each other with a phase shift in each configuration. However, the dynamics of the all to all configuration is phase shifted from that of the bipartite one even when they start from the same initial conditions. In the network of 3 systems the configurations 3 and 4 exhibited frequency synchronized states with no clusters. In the network of 4 systems with ring topology, in configuration 4 , the two fast systems directly connected to slow form a cluster. Similar behavior is seen in configuration 6 where the two slow systems directly connected to fast form a cluster. In configuration 5, we see clustering of slow and fast systems.

\section{Conclusion}

Our study shows how mismatch in time scales of different interacting units affects the emergent collective dynamics of the whole system. In an all to all connected network of $N$ systems with $m$ of them having dynamics of slower time scale, the systems can, in general, separate into two clusters, one of slow systems and the other of fast systems, each having synchronized dynamics among its units.

The nature of dynamics of each cluster depends on the strength of coupling $\epsilon$ and the time-scale mismatch $\tau$. The cases where the two clusters settle to frequency synchronization between them and that for which the whole network stabilizes to amplitude death are studied in particular and the transitions between them identified.

We report an interesting crossover behavior in both the frequency of the emergent state as well as in the emergent dynamics as the number of slow systems increases. The crossover points in both cases are studied for different values of time-scale mismatch.

We study in detail the dynamics in minimal networks of 3 and 4 systems. By computing the eigenvalues of the Jacobian we identify configurations having similar transitions to AD. We also study the similarity in the dynamics outside the $\mathrm{AD}$ region among all the possible configurations. These can serve as substructures or motifs in much larger networks under similar situations.

It is interesting to note that occurrence of the various phenomena observed in the study, like frequency synchronized clusters, frequency suppressed states, amplitude death can be controlled by tuning the parameters $m, \epsilon$ and $\tau$. This means that one can achieve desired frequency in the output of the whole network by a suitable choice of these parameters. This can be a possible process that occurs in real-world networks like neurons. At the same time it can have engineering applications in power transmission networks, traffic systems and sensor networks where controlled frequencies play a relevant role.

\section{Acknowledgements}

KG would like to thank University Grants Commission, New Delhi, for financial support.

\section{References}

[1] A Arenas, A Diaz-Guilera, J Kurths, Y Moreno and C Zhou, Phys. Rep. 469, 93 (2008)

[2] V Resmi, G Ambika, R E Amritkar and G Rangarajan, Phys. Rev. E 85, 046211 (2012)

[3] Y Zhu, Z Zheng and J Yang, Phys. Rev. E 89, 022914 (2014)

[4] E Schöll, Eur. Phys. J. Spec. Top. 225, 891 (2016)

[5] R Kong, T Wu, Y-Ying Qiu and L Wang, Phy. Lett. A 378, 950 (2014)

[6] C Parra-Rojas, T House and A J MacKane, Phys. Rev. E 94, 062408

[7] W Feng and K Takemoto, Sci. Rep. 4, 5912 (2014)

[8] S Tan and J Lü, Sci. Rep. 4, 5034 (2014)

[9] J Law, Systems Practice 5, 379 (Springer, 1992) 
[10] M Denker, M Timme, M Diesmann, F Wolf and T Geisel, Phys. Rev. Lett. 92, 074103 (2004)

[11] A E Motter and Y-C Lai, Phys Rev. E. 66, 065102(R) (2002)

[12] D Das and D S Ray, Eur. Phys. J. Special Topics 222, 785 (2013)

[13] M C Soriano, L Zunino, O A Rosso, I Fischer and C R Mirasso, IEEE J. Quantum Electron. 47, 252 (2011)

[14] G D Mitsis, R Zhang, B D Levine and V Z Marmarelis, Annals of Biomedical Eng. 30, 555 (2002)

[15] L Kay, Chaos 13, 1057 (2001)

[16] J David Neelin, J. Atmos. Sci. 48, 584 (1991)

[17] B R lindner and J D Neelin, J. Climate 21, 2187 (2008)
[18] K D Williams, W J Ingram and J M Gregory, J. Climate 21, 5076 (2008)

[19] G Meehl, et al., Cli. Dyn. 17, 753 (2001)

[20] S De Monte, F d'Ovidio and E Mosekilde, Phys. Rev. Lett. 90, 054102 (2003)

[21] K Fujimoto and K Kaneko, Physica D. 180, 1 (2003)

[22] K Gupta and G Ambika, Eur. Phys. J. B 89, 147 (2016)

[23] R Milo, S Shen-Orr, S Itzkovitz, N Kashtan, D Chklovskii and U Alon, Science 298, 824 (2010)

[24] C-Y Huang, C-T Sun, C-Y Cheng and J-L Hsieh, Physica A 377, 340 (2007)

[25] M Salehi, H R Rabiee and M Jalili, Physica A 389, 5521 (2010) 